\title{
Progression of LSIL to HSIL or SCC: is anoscopy and biopsy good enough?
}

\author{
M. Mistrangelo ${ }^{1} \cdot$ A. Salzano ${ }^{1}$
}

Received: 7 May 2019 / Accepted: 8 May 2019 / Published online: 18 May 2019

(c) Springer Nature Switzerland AG 2019

Persistent human papillomavirus (HPV) infection is strongly associated with the development of anal cancer ( $80 \%$ of cases).

The incidence of squamous cell carcinoma (SCCA) of the anus has been increasing in the past decade, and occurs at higher rates in homosexual men (MSM) with human immunodeficiency virus (HIV) infection.

High-grade anal intraepithelial neoplasia (HSIL) is believed to be the direct precursor to anal cancer.

The rate of progression of HSIL to invasive carcinoma is $1.3 \%$ and $3.2 \%$ at 5 years in two recent reviews $[1,2]$. In a meta-analysis progression rates from AIN III to anal cancer are approximately 1 in 600 per year in HIV-positive MSM and 1 in 4000 per year in HIV-negative MSM patients [2].

More recently Arens et al. reported that the cumulative incidence of SCCA after a diagnosis of AIN III was $1.2 \%$ at 12 months; $2.6 \%$ at 24 months; $3.7 \%$ at 36 months, and $5.7 \%$ at 60 months [3]. On adjusted analysis, Arens and colleagues were not able to detect any statistically significant predictors of progression to SCCA, even though a history of ano-genital condylomata was associated with an increased risk of progression to SCCA [3].

As far as disease progression in HIV-infected MSM, the best data is that of Burgos et al. who reported that the cumulative incidence of AIN III was $7.2 \%$ at 12 months, $16.2 \%$ at 24 months and $24.5 \%$ at 36 months [4]. Considering these data, it is widely accepted that participation in an anal cancer screening programs can lead to detection and treatment of HSIL, decreasing the risk of progression to SCCA [3].

However there are no universal recommendations for the screening and management of HSIL. Proposals for anal cancer screening in MSM are based largely on the model of cervical cancer screening. These programmes recommend

M. Mistrangelo

mistrangelo@katamail.com

1 Surgical Science Department, Città della Salute e della Scienza Hospital, Turin, Italy performing anal cytology and high-resolution anoscopy (HRA) annually and more frequently if any abnormality is observed. However, the scientific evidence for this advice is limited and anal cancer screening programs targeting highrisk populations have been implemented to prevent SCCA by finding and treating HSIL, although the benefits of screening are still controversial and due to the low rate of progression screening is unlikely to be cost effective.

Current techniques for detecting anal cancer precursors include cytology and high-risk human papillomavirus (hrHPV) detection, digital anorectal examination (DARE) and high resolution anoscopy (HRA). DARE is mandatory for all high-risk patients. In the majority of cases DARE findings are sufficient to arouse suspicion of anal cancer. In the general population, sensitivity of cytology for anal HSIL is poor due to low prevalence and the reverse is true in highrisk groups. Cuming and Nathan reviewed the results of anal cytology reporting a sensitivity between 54 and $89 \%$ with a specificity between 37 and $76 \%$ [5]. The association of HPV testing and cytology in HIV negative patients improves sensitivity. The negative predictive value of combining the tests is $93 \%[5,6]$.

In patients with abnormal cytology, high-resolution anoscopy-guided biopsy should be used to identify dysplastic lesions. Though HRA is similar to colposcopy, specific training is required to become an expert in the technique. At present, the lack of experts in HRA limits the creation of new screening programs.

Nowadays HRA is considered the best diagnostic tool in the detection of anal cancer precursors mainly in high-risk populations (MSM HIV + patients) in which HSIL prevalence is $25-35 \%$. European guidelines for the management of AIN, with preventive intent, are based on the following triad: detection by cytology, lesion identification by HRA, and confirmation by HRA- guided biopsy [7].

However the Clinical Practice Guidelines of the American Society of Colorectal Surgeons (ASCRS) report that there is a weak recommendation based on moderate-quality 
evidence, $2 \mathrm{~B}$, for all the above-mentioned diagnostic tools: screening with anal cytology, HPV testing and HRA [8].

The obvious goal of anal cancer screening is to identify and treat both early invasive anal SCCA and high-grade dysplasia (HSIL). The removal of HSIL is thought to prevent progression to anal cancer in almost all patients. However, the recurrence rate of HSIL is high. HRA with targeted biopsy and ablation should be considered the standard of care for patients diagnosed with AIN, with follow-up every 3-4 months for repeat evaluations until the disease is no longer present [9].

In this month's issue of Techniques in Coloproctology, McCutcheon and colleagues [10] published a paper regarding predisposing factors of progression of anal intraepithelial neoplasia in $161 \mathrm{HIV}-1$ positive individuals. They report that HRA was not utilized, since this procedure is not part of an institutional protocol. Moreover, referring to the ASCRS guidelines, they suggest that there has been no definitive data that has suggested HRA is superior to expectant management with close observation in the prevention of anal cancer. In their series progression of AIN was observed in $9 \%$ of patients, while 2 patients out of $161(1.2 \%)$ within a mean of 331 days progressed to cancer. The progression to SCCA is similar to that of the HRA literature at 1 year, and it is expected to rise with longer follow-up. Regarding disease progression, the numbers are also similar to those reported in the literature although underdiagnosis of disease progression is possible if HRA is not used. The median follow-up of 331 days is very short, since, as highlighted above, the incidence increases with the length of follow-up. It would have been interesting to know how many patients were on highly active antiretroviral therapy (HAART), although we suspect that the majority was, give that $85 \%$ had an undetectable viral load. Use of HAART has been reported to slow the progression of AIN to SCCA and may in fact change what we know about this disease [2]. Interestingly, the authors have found a protective effect of anal cytology which suggests a possible role of serial cytology in controlling disease progression. A history of condyloma and a low CD4 count increased disease progression, as previously reported in the literature, while, surprisingly, the presence of high-risk serotypes was associated with disease stability or regression.

When HRA is not performed, a follow-up plan for AIN including multiple random biopsies should be instituted. Standard anoscopy may only reveal the most advanced cases of AIN or anal cancer.

In conclusion, the exact natural history of the progression of AIN and the benefits of performing HRA are still uncertain and results of ongoing trials are awaited. In this context, it is important to report the results when these high-risk patients are only diagnosed and followed up with anoscopy, as this reflects the practice in the real world in the age of HAART. HRA is not commonly performed due to skepticism or lack of resources. Better studies with longer follow-up will reveal if this skepticism is justified and if HRA is a cost effective preventive measure.

\section{Compliance with ethical standards}

Conflict of interest The authors declare that they have no conflict of interest.

Ethical approval This article does not contain any studies with human participants or animals performed by any of the authors.

Informed consent For this type of study formal consent is not required.

\section{References}

1. Cachay ER, Mathews WC (2013) Human papillomavirus, anal cancer, and screening considerations among HIV-infected individuals. AIDS Rev 15:122-133

2. Machalek DA, Poynten M, Jin F, Fairley CK, Farnsworth A, Garland SM, Hillman RJ, Petoumenos K, Roberts J, Tabrizi SN et al (2012) Anal human papillomavirus infection and associated neoplastic lesions in men who have sex with men: a systematic review and meta-analysis. Lancet Oncol 13(487-500):31

3. Arens Y, Gaisa M, Goldstone S, Liu Y, Wisnivesky J, Sigel C, Swartz T, Sigel K (2019) Risk of invasive anal cancer in HIVinfected patients with high-grade anal dysplasia: a population-based cohort study. Dis Colon Rectum. https://doi.org/10.1097/dcr.00000 0000000138

4. Burgos J, Currana A, Talladab N, Guelarc A, Navarroa J, Landolfi S, Villarc J, Crespoa M, Riberaa E, Falco V (2015) Risk of progression to high-grade anal intraepithelial neoplasia in HIV-infected MSM. AIDS 29:695-702

5. Cuming T, Nathan M (2017) Anal cancer screening: techniques and guidelines. Semin Colon Rectal Surg 28:69-74

6. Berry JM, Palefsky JM, Jay N, Cheng SC, Darragh TM, Chin-Hong PV (2009) Performance characteristics of anal cytology and human papilloma virus testing in patients with high-resolution anoscopyguided biopsy of high-grade anal intraepithelial neoplasia. Dis Colon Rectum 52(2):239-247

7. European AIDS Clinical Society Guidelines Version 8.1 (2016) https ://eacsociety.org/files/guidelines_8.1-english.pdf. Accessed $28 \mathrm{Feb}$ 2017

8. Stewart DB, Gaertner WB, Glasgow SC, Herzig DO, Feingold D, Steele SR (2018) The American Society of Colon and Rectal Surgeons Clinical Practice Guidelines for Anal Squamous Cell Cancers (Revised 2018). Dis Colon Rectum 61:755-774. https://doi. org/10.1097/DCR.0000000000001114

9. Messick CA, Rodriguez-Bigas MA (2017) Anal Dysplasia Surg Oncol Clin N Am 26:33-43

10. McCutcheon T, Hawkins AT, Muldoon RL, Hopkins MB, Geiger TM, Ford MM (2019) Progression of anal intraepithelial neoplasia in HIV-positive individuals: predisposing factors. Tech Coloproctol. https://doi.org/10.1007/s10151-019-01951-w (Epub ahead of print)

Publisher's Note Springer Nature remains neutral with regard to jurisdictional claims in published maps and institutional affiliations. 